\title{
A Double Power Reaching Law of Sliding Mode Control Based on Neural Network
}

\author{
Yu-Xin Zhao, Tian Wu, and Yan Ma \\ College of Automation, Harbin Engineering University, Harbin 150001, China \\ Correspondence should be addressed to Yu-Xin Zhao; zhaoyuxin@hrbeu.edu.cn
}

Received 21 July 2013; Accepted 19 August 2013

Academic Editor: Rongni Yang

Copyright (C) 2013 Yu-Xin Zhao et al. This is an open access article distributed under the Creative Commons Attribution License, which permits unrestricted use, distribution, and reproduction in any medium, provided the original work is properly cited.

For discrete system, the reaching law election and controller design are two crucial and important problems. In this paper, an improved double power reaching law of SMC and a controller combined with neural network have been investigated. Theory proves that this method can eliminate the chattering and increase the reaching rate. Furthermore, when there is a certain external interference, the regulating function of neural network can ensure strong robustness of the system. Simulation results show that compared with exponential reaching law, single power reaching law, and traditional double power reaching law, the proposed reaching law has faster convergence speed and better dynamic performance.

\section{Introduction}

Sliding mode variable structure control theory is essentially a kind of special nonlinear control, and its nonlinear performance is the discontinuity for the control [1]. Due to the rapid development of computer technology, discrete-time system is widely used in the actual control; therefore, the sliding mode control for discrete-time system is especially important $[1,2]$. Based on the reaching law, Gao et al. proposed the discrete sliding mode variable structure control [3]. Koshkouei and Zinober proposed the existence conditions of a new sliding mode and designed a new sliding mode control law [4]. But it is difficult to effectively guarantee the robustness of sliding mode control. Chen studied sliding mode control for the multiple input-output discrete-time system with disturbances and unknown parameters, and an adaptive law was implemented to estimate the unknown term [5].

Artificial neural network has strong learning ability and high ability of parallel computing, and it can approximate any nonlinear function and has good robustness and fault tolerance $[6,7]$. In recent years, the combination of neural network with sliding mode variable structure control method [8-10] has become a new field for variable structure development. The nonlinear part of neural network, uncertainties, and unknown external disturbance were estimated online for the linear system, and the equivalent control was realized based on the neural network, and the chattering was eliminated effectively [11]. Ertugrul and Kaynak proposed a new sliding mode control method based on neural network, which used two neural networks to approximate equivalent sliding mode control and the part of switching sliding mode control, without object model, and it effectively eliminated the chattering [12]. Huang et al. designed a sliding mode controller by using the approximation ability of RBF neural network; the switching function was regarded as the input of the network, the controller was completely realized by continuous RBF function, and this method cancelled the switching part and eliminated the chattering [13]. Da divided the sliding mode controller into two parts: one part was the neural network sliding mode controller and the other part was a linear feedback controller, and it replaced the switching function with the output of fuzzy neural network, which ensured the continuity of the control law and fundamentally eliminated chattering [14]. A radial basis function (RBF) neural network controller based on Lyapunov theory was designed to compensate for the unknown nonlinearity in the system, and the neural network controller guaranteed the stability of the closedloop system, and simulation results showed the desirable performances of the presented controller in the early phase of operation and in the strong disturbance situation [15].

In all kinds of nonlinear systems, the chattering is caused by the influence of time delay and inertia in time or space, 
which affects the accuracy of control system. It may also stimulate strong oscillation of the unmodelled part and cause harm to the system [2]. Therefore, the suppression of chattering became an important subject in the field of sliding mode variable structure control. Wu et al. introduced some slack matrices and a delay-dependent sufficient condition to guarantee the sliding mode dynamics, and this method is to be generalized quadratically stable and robustly passive $[16,17]$. And $\mathrm{Wu}$ et al. designed an integral sliding surface function and an observer to estimate the system states, which was proved to be attained in a finite time $[18,19]$. An integral type sliding surface function is designed for establishing a sliding mode dynamics, which can be formulated by a switched stochastic system with an external disturbance/uncertainty [20].

In recent years, the power reaching law has become the focus of attention. For the deterministic system, Ren et al. derived a quasisliding mode bandwidth for the power reaching law [21], Mi et al. further presented the arrival time [22]. Sun et al. constructed the discrete reaching law by using the power function and made an analysis of the convergence for the control algorithm [23]. Li et al. discussed continuous forms of the power reaching law and got the steady state error bounds across the sliding mode bandwidth [24]. Mei and Wang proposed a new double power reaching law, which made the system have a higher speed in the whole process of reaching sliding surface, and eliminate the inherent chattering of the sliding mode variable structure in traditional control [25]. Li et al. proposed a new double power reaching law based on the analysis of the common reaching law, and this new law could not only guarantee the fast reaching movements, but also greatly weakened the chattering and improved the anti-interference ability [26]. Zhang et al. proposed a design scheme which used a double power reaching law to improve the convergence speed in both away from and near the sliding mode space [27].

This paper is organized as follows. The next content consists of five sections about reaching law selection and controller design. The state equation is described in Section 2, and a double power reaching law is proposed and an explanation on the reaching law is made. Section 3 gives the reaching condition and analyses the stability and reaching rate, and the rationality for the parameters setting is described. Section 4 designs a controller combined with neural network and gives the structure of RBF network. Section 5 shows that compared with exponential reaching law, single power reaching law, and traditional double power reaching law, the proposed reaching law has faster convergence speed and better dynamic performance. Finally, we conclude this paper in Section 6.

\section{Problem Description and Preliminaries}

For the discrete-time uncertain system, the state equation can be expressed as follows:

$$
x(k+1)=A x(k)+B u(k)+f(k),
$$

where $x(k) \in R^{n}$ is a state vector, $u(k) \in R$ is the control input, $A \in R^{n \times n}$ is a constant matrix, $B \in R^{n}$ is a constant vector, and $f(k) \in R^{l}$ is external interference, which generally cannot be measured.

To analyse the tracking problem of discrete system, firstly, we should get the error state equation. Assuming that $R(k)$ is the command signal of hope, and $x_{e}(k)=e(k)=R(k)-$ $x(k)$. We can get the discrete error equation of state across the system (1). Consider

$$
x_{e}(k+1)=A_{e} x_{e}(k)+B_{e} u(k)+f_{e}(k),
$$

where $A_{e}=A, B_{e}=-B, f_{e}(k)=-A R(k)+R(k+1)-f(k)$, $R(k)=[r(k), d r(k)]^{T}, d r(k)$ is the change rate of position, $x_{e}(k)=[e(k), d e(k)], e(k)$ is error, and $d e(k)$ is the change rate of error.

Select the switch function

$$
s(k)=C_{e} x_{e}(k),
$$

where $C_{e}$ is the constant matrix needed to design, and we should guarantee $C_{e} B_{e} \neq 0$.

In the sliding mode variable structure control system, movement of the system can be divided into two stages, respectively, reaching stage and sliding motion stage. The process of system moves to the switching surface from any initial state is called reaching movement, namely, the reaching process of $s \rightarrow 0$ [28]. And the sliding motion is the movement on the surface of sliding mode $s=0$.

Power reaching law is a kind of common reaching law, and its approaching speed decreases with distance when the system state is close to the sliding mode plane, which is benefit for weakening the chattering. But when the system state is away from the sliding mode plane, there exists a problem that the approaching speed is too small to affect the performance of system [29], which makes the reaching time too long.

This paper aims at the improvement of the power reaching law on the basis of guaranteeing its original merits and improving its reaching performance when the system state is away from the sliding mode plane. The double power-law exponent reaching law expression is as follows:

$$
\begin{aligned}
s(k+1)-s(k)= & -q T s(k)-\varepsilon_{1} T|s(k)|^{\alpha} \operatorname{sgn}(s(k)) \\
& -\varepsilon_{2} T|s(k)|^{\beta} \operatorname{sgn}(s(k)),
\end{aligned}
$$

where $\varepsilon_{1}>0, \varepsilon_{2}>0, q>0,1-q T>0,0<\alpha<1, \beta>1, T$ is the sampling time.

In expression (4), when $|s(k)|<1$, the reduction of value $\alpha$ can increase the approaching speed, and when $|s(k)|>1$ and $\alpha$ decrease, the impact of $-\varepsilon_{1} T|s(k)|^{\alpha} \operatorname{sgn}(s(k))$ reduces, and compared with $-\varepsilon_{2} T|s(k)|^{\beta} \operatorname{sgn}(s(k))$, it can be neglected. When $|s(k)|>1$, the increase of value $\beta$ can enlarge the approaching speed, and when $|s(k)|<1$ and $\beta$ increase, the impact of $-\varepsilon_{2} T|s(k)|^{\beta} \operatorname{sgn}(s(k))$ reduces, and compared with $-\varepsilon_{1} T|s(k)|^{\alpha} \operatorname{sgn}(s(k))$, it can be neglected.

Since the system uses a piecewise control, it is not smooth transition in the boundary, which affects the dynamic quality of the system. In order to overcome the defects, $q T s(k)$ is used to relieve the discontinuity in the demarcation point and make the system chattering attenuation in exponential form; thus, the chattering is weakened and the system is guaranteed 
to be stable. Expression (4) shows that when $s(k)=0, s(k+$ $1)=0$. That is to say, the system can realize smooth transition to reach the sliding surface.

\section{System Analysis and Proof}

In order to make the system meet the performance requirements, we should analyze the system from the following aspects.

3.1. Existence and Reaching Condition. Based on the discrete sliding mode theory $[30,31]$, a discrete system should meet the following conditions to guarantee the existence and the reaching condition of sliding mode:

$$
\begin{aligned}
& {[s(k+1)-s(k)] \operatorname{sgn}(s(k))<0,} \\
& {[s(k+1)+s(k)] \operatorname{sgn}(s(k))>0,}
\end{aligned}
$$

where the sampling time $T$ is very small.

Using expression (4) to system (2), we can get the following function:

$$
\begin{aligned}
& {[s(k+1)-s(k)] \operatorname{sgn}(s(k))} \\
& =\left[-q T s(k)-\varepsilon_{1} T|s(k)|^{\alpha} \operatorname{sgn}(s(k))\right. \\
& \left.\quad-\varepsilon_{2} T|s(k)|^{\beta} \operatorname{sgn}(s(k))\right] \operatorname{sgn}(s(k)) \\
& =-q T|s(k)|-\varepsilon_{1} T|s(k)|^{\alpha}-\varepsilon_{2} T|s(k)|^{\beta}<0,
\end{aligned}
$$

and when the sampling time $T$ is very small, $(2-q T) \gg 0$.

And the following expression can be obtained:

$$
\begin{aligned}
& {[s(k+1)+s(k)] \operatorname{sgn}(s(k))} \\
& =\left[(2-q T) s(k)-\varepsilon_{1} T|s(k)|^{\alpha} \operatorname{sgn}(s(k))\right. \\
& \left.\quad-\varepsilon_{2} T|s(k)|^{\beta} \operatorname{sgn}(s(k))\right] \operatorname{sgn}(s(k)) \\
& =(2-q T)|s(k)|-\varepsilon_{1} T|s(k)|^{\alpha}-\varepsilon_{2} T|s(k)|^{\beta}>0 .
\end{aligned}
$$

Therefore, the double power-law exponent reaching law (4) can satisfy the reaching condition, which can ensure the good dynamic quality for the system.

3.2. Stability Analysis. Assuming that $\Delta s(k)=s(k+1)-s(k)$, we can get expression (8) from (4):

$$
\begin{aligned}
\Delta s(k)= & -q T s(k)-\varepsilon_{1} T|s(k)|^{\alpha} \operatorname{sgn}(s(k)) \\
& -\varepsilon_{2} T|s(k)|^{\beta} \operatorname{sgn}(s(k)) .
\end{aligned}
$$

(i) When $s(k) \geq 0$,

$$
\Delta s(k)=-q T s(k)-\varepsilon_{1} T[s(k)]^{\alpha}-\varepsilon_{2} T[s(k)]^{\beta} .
$$

The derivation of (9) on both sides is as follows:

$$
\frac{\partial \Delta s(k)}{\partial s(k)}=-q T-\varepsilon_{1} T \alpha[s(k)]^{\alpha-1}-\varepsilon_{2} T \beta[s(k)]^{\beta-1} .
$$

By the setting parameters, we know $\partial \Delta s(k) / \partial s(k)<0$; that is to say, $\Delta s(k)$ is a minus function of $s(k)$ :

$$
\Delta s(k) \leq-q T s(k)-\varepsilon_{1} T[s(k)]^{\alpha}-\left.\varepsilon_{2} T[s(k)]^{\beta}\right|_{s(k)=0}=0 .
$$

Due to $\Delta s(k) \leq 0, s(k)$ declines until it is close to the state $s(k)=0$. And only when $s(k)=0, \Delta s(k)=0$.

(ii) When $s(k)<0$,

$$
\Delta s(k)=-q T s(k)+\varepsilon_{1} T[-s(k)]^{\alpha}+\varepsilon_{2} T[-s(k)]^{\beta} .
$$

The derivation of (12) on both sides is as follows:

$$
\frac{\partial \Delta s(k)}{\partial s(k)}=-q T-\varepsilon_{1} T \alpha[-s(k)]^{\alpha-1}-\varepsilon_{2} T \beta[-s(k)]^{\beta-1} .
$$

By the setting parameters, we know $\partial \Delta s(k) / \partial s(k)<0$; that is to say, $\Delta s(k)$ is a minus function of $s(k)$ :

$$
\Delta s(k)>-q T s(k)-\varepsilon_{1} T[s(k)]^{\alpha}-\left.\varepsilon_{2} T[s(k)]^{\beta}\right|_{s(k)=0}=0 .
$$

Due to $\Delta s(k)>0, s(k)$ increases until it is close to the state $s(k)=0$.

(iii) When $s(k)=0$,

$$
s(k+1)=s(k)=0 .
$$

It is easy to know that the system enters into a stable state this moment.

According to the analysis above, in the process of sliding mode motion, the value of $|s(k)|$ is close to 0 infinitely. Thus, the stability of system is fine, and there is no chattering in theory.

3.3. Reaching Rate Analysis. A good reaching law should ensure that the system reaches the sliding surface steadily and speedily. And in order to achieve a smooth transition, the rate in the demarcation point should be commanded to be as small as possible, which can restrain the chattering.

From expression (4), we can see that $-\varepsilon_{1} T|s(k)|^{\alpha} \operatorname{sgn}(s(k))$ ensures the dynamic quality when system is close to the sliding mode plane $(|s(k)|<1),-\varepsilon_{2} T|s(k)|^{\beta} \operatorname{sgn}(s(k))$ ensures the dynamic quality when system is far away from the sliding mode plane $(|s(k)|>1)$, and $-q T s(k)$ ensures the smooth transition in the demarcation point. The combination of the three above makes the system reach the sliding surface quickly and smoothly from any initial state.

Assume that initial state $s(0)>1$, and the process of reaching the sliding surface $s(k)=0$ from initial state can be divided into two stages, from the initial state to $s(k)=1$ and from $s(k)=1$ to the sliding mode $s(k)=0$.

(i) From $s(0)$ to $s(k)=1$.

At this point $s(k) \geq 1$. In expression (4), $0<$ $\alpha<1, \beta>1$, and the role of $-\varepsilon_{2} T|s(k)|^{\beta} \operatorname{sgn}(s(k))$ is far more than $-\varepsilon_{1} T|s(k)|^{\alpha} \operatorname{sgn}(s(k))$. So the influence of $-\varepsilon_{1} T|s(k)|^{\alpha} \operatorname{sgn}(s(k))$ can be ignored. We can get the following function:

$$
s(k+1)-s(k)=-q T s(k)-\varepsilon_{2} T[s(k)]^{\beta} .
$$


If $T$ is small enough, the derivative of continuous system is defined as follows:

$$
\dot{s}(t)=\lim _{\Delta t \rightarrow 0} \frac{s(t+\Delta t)-s(t)}{\Delta t} .
$$

Therefore, $\dot{s}=(s(k+1)-s(k)) / T=-q s-\varepsilon_{2} s^{\beta}$ is available.

Computing the time of arrival for system at this state, the following equation can be obtained:

$$
\frac{d s}{d t}+q s=-\varepsilon_{2} s^{\beta}
$$

both sides multiplied by $s^{-\beta}$

$$
s^{-\beta} \frac{d s}{d t}+q s^{1-\beta}=-\varepsilon_{2} .
$$

Assuming that $y=s^{1-\beta}$

$$
\frac{d y}{d t}=(1-\beta) s^{-\beta} \frac{d s}{d t},
$$

both sides of (19) are multiplied by $(1-\beta)$

$$
(1-\beta) s^{-\beta} \frac{d s}{d t}+(1-\beta) q s^{1-\beta}=-(1-\beta) \varepsilon_{2} .
$$
(21):

Equation (22) is obtained by the combination of (20) and

$$
\frac{d y}{d t}+(1-\beta) q y=-(1-\beta) \varepsilon_{2}
$$

And for the first order linear nonhomogeneous differential equation

$$
\frac{d y}{d t}+P(t) y=Q(t)
$$

its general solution form [32] is as follows:

$$
y=c e^{-\int P(t) d t}+e^{-\int P(t) d t} \int Q(t) e^{\int P(t) d t} d t .
$$

The constant $c$ is obtained by the initial value $\left.y\right|_{t=0}$.

Combined with (24), the solution for (22) is available as follows:

$$
y=c e^{-\int(1-\beta) q d t}+e^{-\int(1-\beta) q d t} \int\left[-(1-\beta) \varepsilon_{2}\right] e^{\int(1-\beta) q d t} d t
$$

Calculating formula (25), we can get

$$
\begin{aligned}
y & =c e^{-(1-\beta) q t}+e^{-(1-\beta) q t} \frac{\left[-(1-\beta) \varepsilon_{2}\right] e^{(1-\beta) q t}}{(1-\beta) q} \\
& =c e^{-(1-\beta) q t}-\frac{\varepsilon_{2}}{q},
\end{aligned}
$$

because of $y=s^{1-\beta}$, so

$$
s^{1-\beta}=c e^{-(1-\beta) q t}-\frac{\varepsilon_{2}}{q} .
$$

When $t=0, s=s(0)$

$$
c=s(0)^{1-\beta}+\frac{\varepsilon_{2}}{q},
$$

we can get (29) by willing (28) into (27).

$$
s^{1-\beta}=\left[s(0)^{1-\beta}+\frac{\varepsilon_{2}}{q}\right] e^{-(1-\beta) q t}-\frac{\varepsilon_{2}}{q} .
$$

The relationship between time $t$ and system state $s$ can be obtained by (29) as follows:

$$
t=\frac{1}{(\beta-1) q}\left\{\ln \left[s^{1-\beta}+\frac{\varepsilon_{2}}{q}\right]-\ln \left[s(0)^{1-\beta}+\frac{\varepsilon_{2}}{q}\right]\right\} .
$$

The time from the initial state $s(0)$ to $s=1$ is

$$
\begin{aligned}
t_{1} & =\frac{1}{(\beta-1) q}\left\{\ln \left[1+\frac{\varepsilon_{2}}{q}\right]-\ln \left[s(0)^{1-\beta}+\frac{\varepsilon_{2}}{q}\right]\right\} \\
& =\frac{\ln \left[1+\left(\varepsilon_{2} / q\right)\right]-\ln \left[s(0)^{1-\beta}+\left(\varepsilon_{2} / q\right)\right]}{(\beta-1) q} .
\end{aligned}
$$

As formula (31) shows, the discrete arrived points for reaching $s(k)=1$ is

$$
k_{1}=\frac{t_{1}}{T}=\frac{\ln \left(q+\varepsilon_{2}\right)-\ln \left[q s(0)^{1-\beta}+\varepsilon_{2}\right]}{(\beta-1) q T} .
$$

At this time, in order to meet the rapidity, we should make the discrete point as small as possible. From (32), we can see that parameters $q, \varepsilon_{2}$, and $\beta$ all affect the reaching speed. When $s(0)>1, q$ gets bigger, $k_{1}$ gets smaller, especially when $q$ is equal to $1 / T$, and the approaching speed is the fastest. The gain parameter $\varepsilon_{2}$ gets bigger and $k_{1}$ gets smaller. The power coefficient $\beta$ gets close to $1, k_{1}$ gets smaller.

(ii) From $s(k)=1$ to $s(k)=0$.

At this point $s(k)<1$. As in expression (4), $0<$ $\alpha<1, \beta>1$, the role of $-\varepsilon_{1} T|s(k)|^{\alpha} \operatorname{sgn}(s(k))$ is far more than $-\varepsilon_{2} T|s(k)|^{\beta} \operatorname{sgn}(s(k))$. So the influence of $-\varepsilon_{2} T|s(k)|^{\beta} \operatorname{sgn}(s(k))$ can be ignored. We can get the following function:

$$
s(k+1)-s(k)=-q T s(k)-\varepsilon_{1} T[s(k)]^{\alpha} .
$$

The specific derivation process of time is as the same as (i) above; we can get the following function:

$$
s^{1-\alpha}=c e^{-(1-\alpha) q t}-\frac{\varepsilon_{1}}{q} .
$$

When $t=0, s=1$

$$
c=1+\frac{\varepsilon_{1}}{q}
$$

we can get (36) by willing (35) into (34) as follows:

$$
s^{1-\alpha}=\left[1+\frac{\varepsilon_{1}}{q}\right] e^{-(1-\alpha) q t}-\frac{\varepsilon_{1}}{q} .
$$




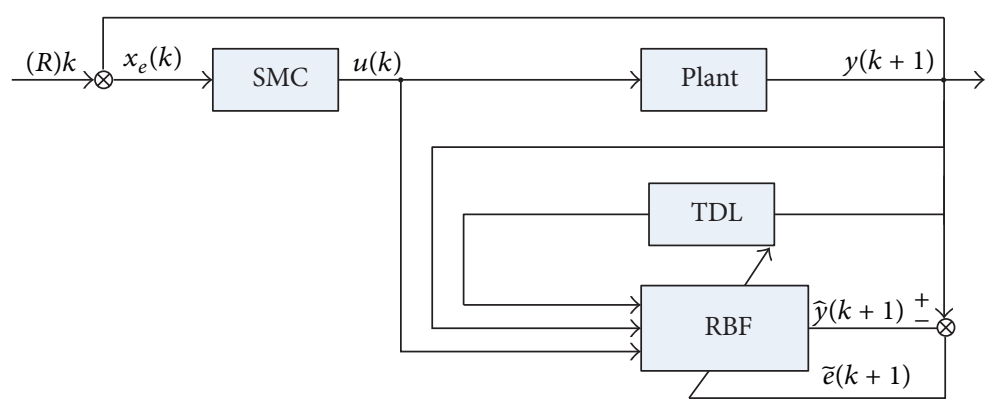

FIGURE 1: The structure for sliding mode control based on neural network.

The relationship between time $t$ and system state $s$ can be obtained by (36) as follows

$$
t=\frac{1}{-(1-\alpha) q}\left\{\ln \left[s^{1-\alpha}+\frac{\varepsilon_{1}}{q}\right]-\ln \left[1+\frac{\varepsilon_{1}}{q}\right]\right\} .
$$

The time from the initial state $s=1$ to $s=0$ is

$$
\begin{aligned}
t_{2} & =\left|\frac{1}{-(1-\alpha) q}\left\{\ln \left[\frac{\varepsilon_{1}}{q}\right]-\ln \left[1+\frac{\varepsilon_{1}}{q}\right]\right\}\right| \\
& =\frac{\ln \left(\varepsilon_{1}+q\right)-\ln \varepsilon_{1}}{(\alpha-1) q} .
\end{aligned}
$$

As formula (38) shows, the discrete arrived points for reaching $s(k)=0$ is

$$
k_{2}=\frac{t_{2}}{T}=\frac{\ln \left(\varepsilon_{1}+q\right)-\ln \varepsilon_{1}}{(\alpha-1) q T} .
$$

At this time, in order to meet the smooth transition, we should make the discrete point $k_{2}$ as large as possible. From (38), we can see that parameters $q, \varepsilon_{1}$, and $\alpha$ all affect the reaching speed. Among them, $q$ gets smaller, $k_{2}$ gets bigger, the gain parameter $\varepsilon_{1}$ gets smaller, $k_{2}$ gets bigger, the power coefficient $\alpha$ gets close to 1 , and $k_{1}$ gets bigger.

From the analysis above, in order to make the system reach the sliding surface rapidity and achieve the smooth transition, and the parameters setting are the key limiting factors. From expressions (32) and (39), we can see that $\varepsilon_{2}$, $\beta$ effect $k_{1}$ mainly and $\varepsilon_{1}, \alpha$ effect $k_{2}$ mainly, but $q$ effect $k_{1}, k_{2}$ at the same time. Since the parameters $\varepsilon_{2}, \beta, \varepsilon_{1}, \alpha$ can be given directly, and the parameter $q$ make an opposite impact on $k_{1}$, $k_{2}$. So in the reaching process, adjusting parameter $q$ to meet the performance requirements is very important, and $s(k)=$ 1 is the dividing point:

$$
q= \begin{cases}q_{\max }, & s(k) \geq 1 \\ q_{\min }, & s(k)<1\end{cases}
$$

where $q_{\max }$ adjusts the dynamic property and $q_{\min }$ adjusts the steady state performance.

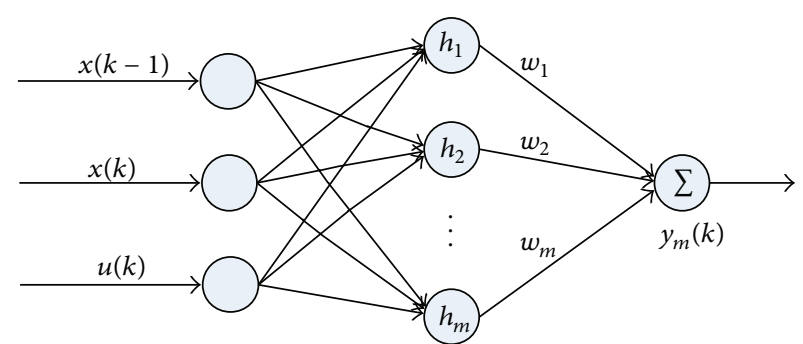

FIGURE 2: The structure for RBF network.

\section{Controller Design}

Combined with the proposed double power-law exponent reaching law (4), we can get the following function for the uncertain system (2):

$$
\begin{aligned}
s(k+1) & \\
= & C_{e} x_{e}(k+1)=C_{e} A_{e} x_{e}(k)+C_{e} B_{e} u(k)+C_{e} f_{e}(k) \\
= & (1-q T) s(k)-\varepsilon_{1} T|s(k)|^{\alpha} \operatorname{sgn}(s(k)) \\
& -\varepsilon_{2} T|s(k)|^{\beta} \operatorname{sgn}(s(k)) ;
\end{aligned}
$$

therefore,

$$
\begin{aligned}
& u(k) \\
& =-\left(C_{e} B_{e}\right)^{-1} \\
& \times\left[C_{e} A_{e} x_{e}(k)-(1-q T) s(k)+\varepsilon_{1} T|s(k)|^{\alpha} \operatorname{sgn}(s(k))\right. \\
& \left.\quad+\varepsilon_{2} T|s(k)|^{\beta} \operatorname{sgn}(s(k))+C_{e} f_{e}(k)\right] .
\end{aligned}
$$

In the actual controller, the external disturbance cannot be measured, which may make the controller a big buffeting. Thus, we use a radial basis function neural network (RBF) to estimate the approximation of unknown part, which is shown in Figure 1. 


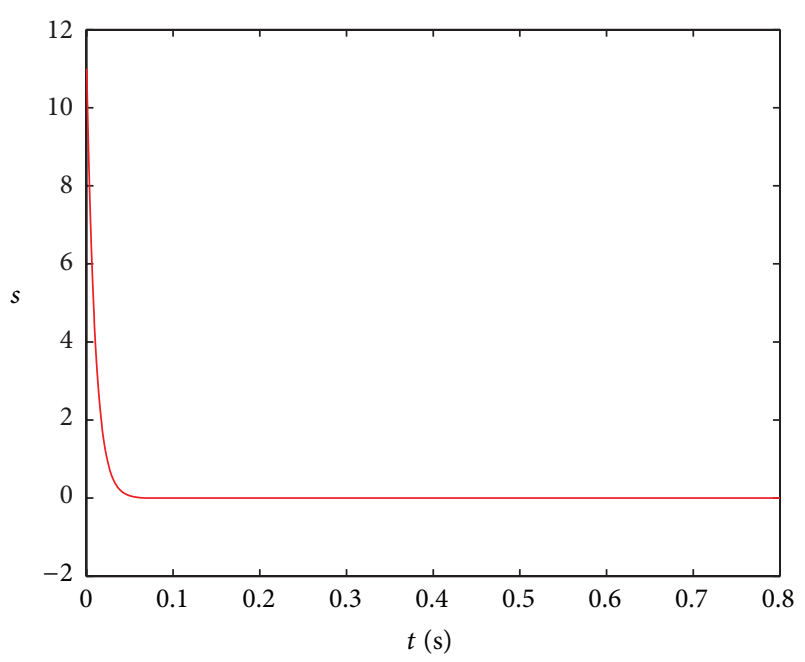

(a)

FIGURE 3: (a) The controller output of reaching law (i).

Here, we use ideal estimate $\widehat{f}_{e}(k)$ to replace $f_{e}(k)$, and the equivalent control law (42) can be written as follows:

$$
\begin{aligned}
& u(k) \\
& =-\left(C_{e} B_{e}\right)^{-1} \\
& \quad \times\left[C_{e} A_{e} x_{e}(k)-(1-q T) s(k)+\varepsilon_{1} T|s(k)|^{\alpha} \operatorname{sgn}(s(k))\right. \\
& \left.\quad+\varepsilon_{2} T|s(k)|^{\beta} \operatorname{sgn}(s(k))+C_{e} \widehat{f}_{e}(k)\right] .
\end{aligned}
$$

The RBF network structure is shown in Figure 2. This network structure includes three output nodes, $m$ hidden nodes, and one output node. Where $X=\left[x_{1}, x_{2}, x_{3}\right]^{T}=$ $[x(k-1), x(k), u(k)]^{T}$ is the input vector. Assume that $H=$ $\left[h_{1} h_{2} \cdots h_{m}\right]^{T}$ is the radial basis vector set of RBF network, where $h_{j}$ is the Koski function.

The output of the RBF networks is expressed as follows:

$$
y_{m}(k)=\widehat{f}(k)=w_{1} h_{1}+w_{2} h_{2}+\cdots+w_{m} h_{m}=\sum_{i=1}^{m} w_{i} h_{i} .
$$

The performance index function of RBF is defined as

$$
E=\frac{1}{2} \sum\left[y(k)-y_{m}(k)\right]^{2}=\frac{1}{2} \sum e^{2}(k) .
$$

According to the gradient descent method, by minimizing the performance index $E$, the learning algorithm weights can be formulated as

$$
\begin{gathered}
\Delta w_{j}=-\frac{\partial E(k)}{\partial w_{j}}=-e(k) \frac{\partial e(k)}{\partial w_{j}}=\left[y(k)-y_{m}(k)\right] h_{j}, \\
w_{j}(k)=w_{j}(k-1)+\eta \Delta w_{j}+\delta\left[w_{j}(k-1)-w_{j}(k-2)\right],
\end{gathered}
$$

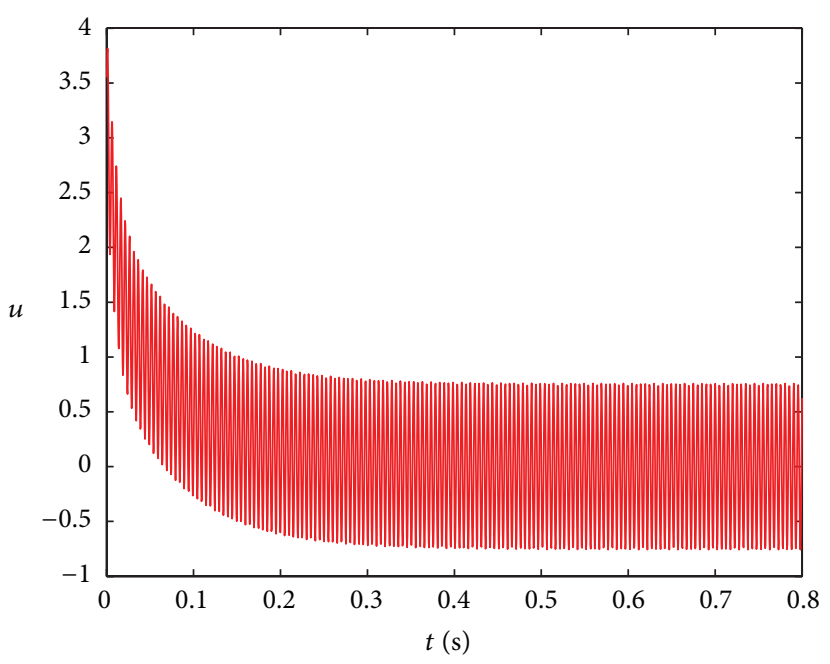

(b)

(b) The switching function curve of reaching law (i).

$$
\begin{aligned}
\Delta b_{j}= & -\frac{\partial E(k)}{\partial b_{j}} \\
= & -e(k) \frac{\partial e(k)}{\partial b_{j}}=\left[y(k)-y_{m}(k)\right] w_{j} h_{j} \frac{\left\|X-c_{j}\right\|^{2}}{b_{j}^{3}}, \\
b_{j}(k) & =b_{j}(k-1)+\eta \Delta b_{j}+\delta\left[b_{j}(k-1)-b_{j}(k-2)\right], \\
\Delta c_{j i} & =-\frac{\partial E(k)}{\partial c_{j i}} \\
& =-e(k) \frac{\partial e(k)}{\partial c_{j i}}=\left[y(k)-y_{m}(k)\right] w_{j} \frac{x_{3 j}-c_{j i}}{b_{j}^{2}}, \\
c_{j i}(k) & =c_{j i}(k-1)+\eta \Delta c_{j i}+\delta\left[c_{j i}(k-1)-c_{j i}(k-2)\right] .
\end{aligned}
$$

Among them, $\eta$ is learning rate, which controls the updating step for parameters, and $\delta$ is momentum factor, which controls the direction of the impact. And $\eta \in(0,1), \delta \in(0,1)$.

\section{Simulation Study}

Consider the following two-order discrete system:

$$
x(k+1)=A x(k)+B u(k)+f(k),
$$

where $A=\left[\begin{array}{ll}1 & 0.001 \\ 0 & 0.9753\end{array}\right], B=\left[\begin{array}{c}-0.0001 \\ -0.1314\end{array}\right]$, and $f(k)=\left[\begin{array}{c}0 \\ 0.1 \sin (k / 2)\end{array}\right]$.

The sampling time $T=0.001 \mathrm{~s}$ and initial state $x(0)=$ $\left[\begin{array}{ll}2 & 1\end{array}\right]^{T}$. The parameters are defined as $c=5, \alpha=0.99, \beta=$ 1.01, $\varepsilon_{1}=1$, and $\varepsilon_{2}=100$. And when $s(k) \geq 1, q=100$, and when $s(k)<1, q=1$. The initial weights of network and Gauss function take random values; the learning parameters take $\delta=0.05, \eta=0.05$.

The switching function takes $s(k)=5 x_{1}(k)+x_{2}(k)$.

Here, we use the following reaching laws to make a comparison with the controlling performance of system. 


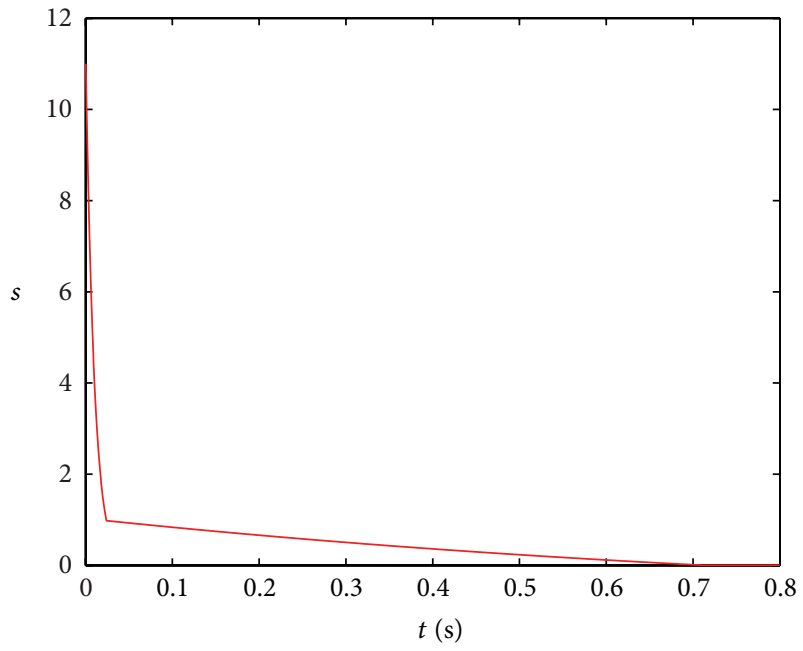

(a)

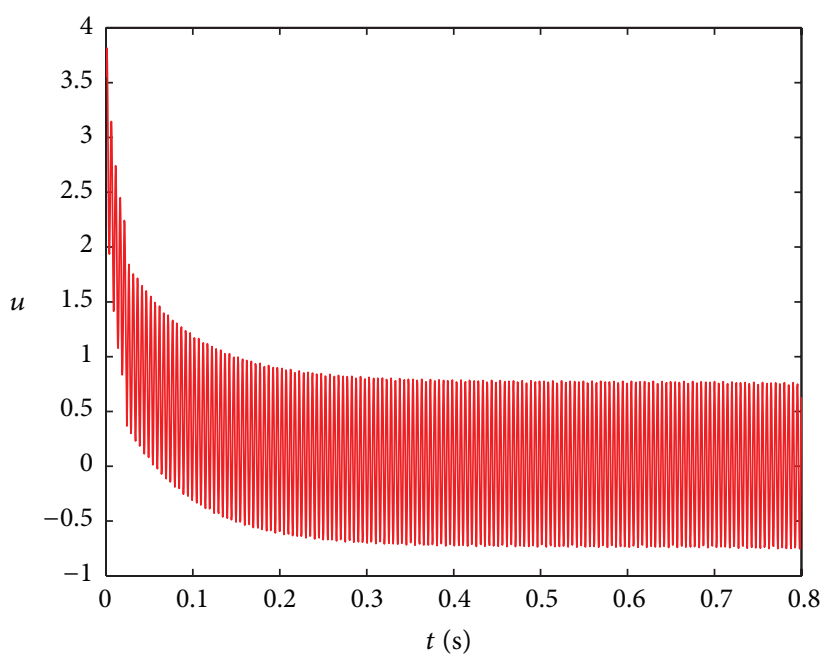

(b)

Figure 4: (a) The controller output of reaching law (ii). (b) The switching function curve of reaching law (ii).

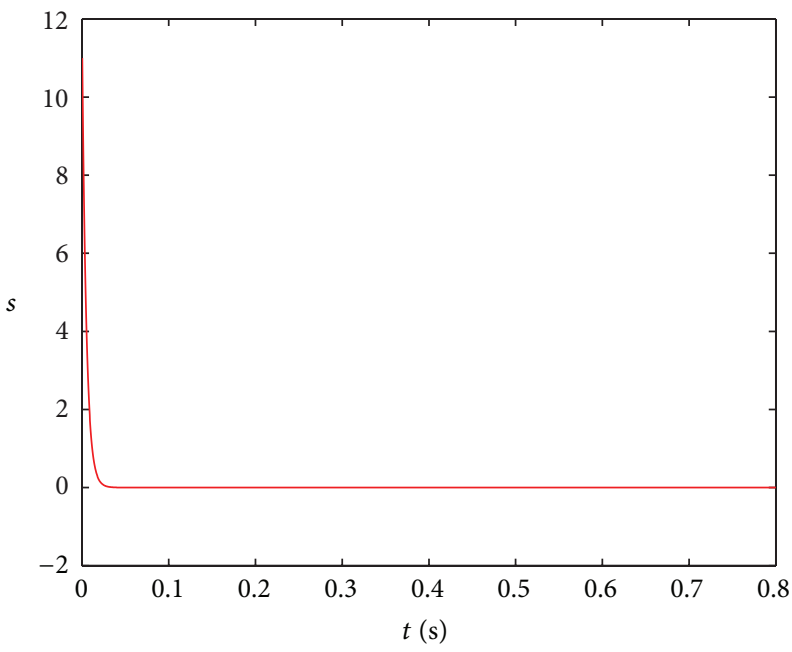

(a)

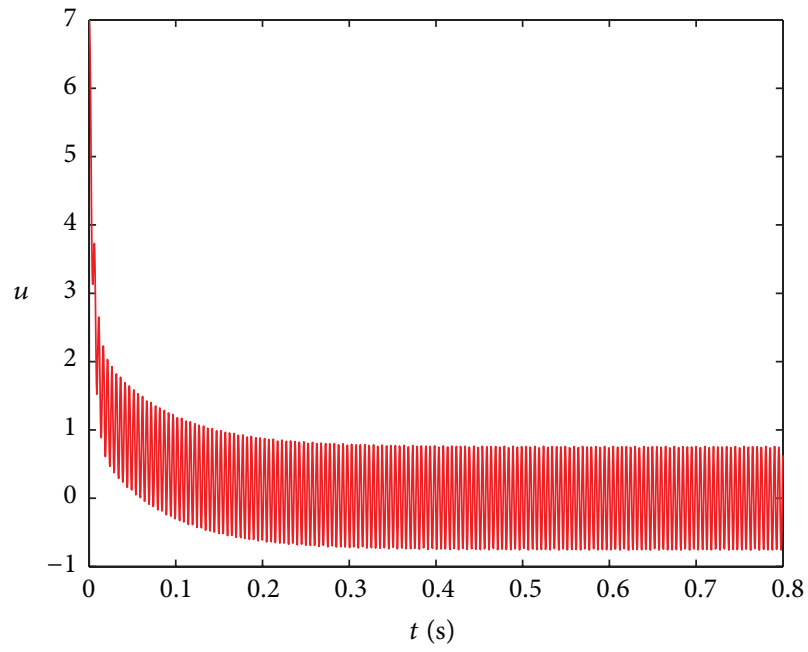

(b)

FIgURE 5: (a) The controller output of reaching law (iii). (b) The switching function curve of reaching law (iii).

(i) Exponential reaching law:

$$
\begin{aligned}
& u_{1}(k) \\
& =-(C B)^{-1}[C A x(k)-(1-q T) s(k) \\
& +\varepsilon T \operatorname{sgn}(s(k))+C f(k)] \text {. }
\end{aligned}
$$

(ii) Single power reaching law:

$$
\begin{aligned}
u_{2}(k)= & -(C B)^{-1} \\
& \times[C A x(k)-(1-q T) s(k) \\
& \left.\quad+\varepsilon T|s(k)|^{\alpha} \operatorname{sgn}(s(k))+C f(k)\right] .
\end{aligned}
$$

(iii) Double power reaching law:

$$
\begin{aligned}
u_{3}(k) & \\
=-(C B)^{-1}[ & C A x(k)-(1-q T) s(k)+\varepsilon_{1} T|s(k)|^{\alpha} \operatorname{sgn}(s(k)) \\
& \left.+\varepsilon_{2} T|s(k)|^{\beta} \operatorname{sgn}(s(k))+C f(k)\right] .
\end{aligned}
$$

(iv) The proposed double power reaching law combined with neural network in this paper.

The controller output and switching function curve of reaching law (i) are shown in Figures 3(a) and 3(b). The controller output and switching function curve of reaching law (ii) are shown in Figures 4(a) and 4(b). The controller output and switching function curve of reaching law (iii) are shown in Figures 5(a) and 5(b). The controller output and switching 


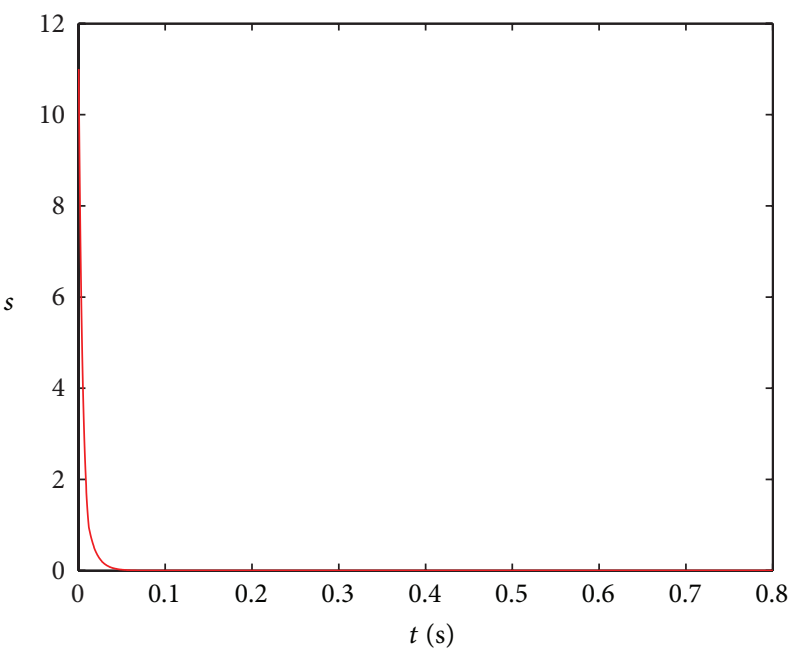

(a)

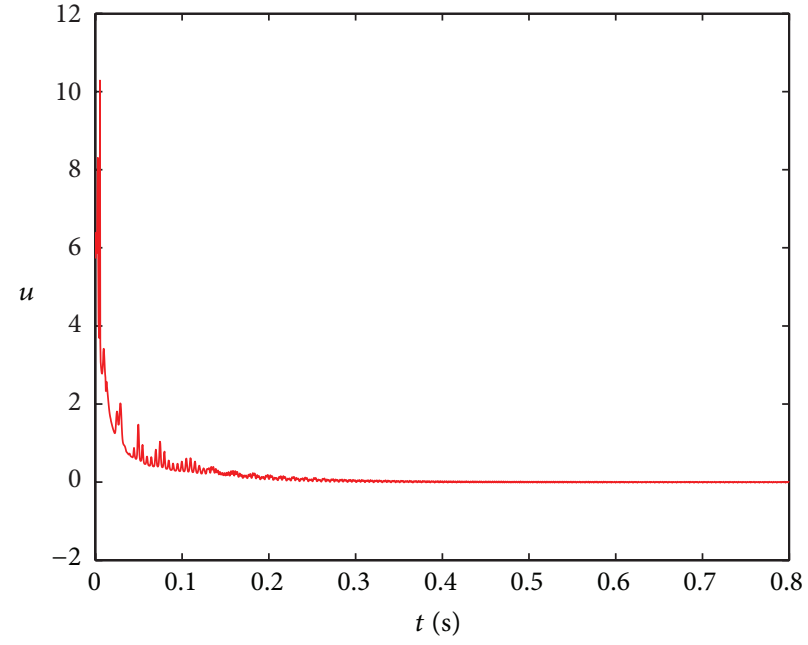

(b)

FIgURE 6: (a) The controller output of reaching law (iv). (b) The switching function curve of reaching law (iv).

function curve of reaching law (iv) are shown in Figures 6(a) and 6(b).

From Figures 3(b), 4(b), and 5(b), we can see that, in certain interference, the controller outputs $u$ of reaching laws (i) (ii) (iii) will produce a severe chattering in the sliding plane. And Figures 3(a), 4(a), and 5(a) show that the system state $s$ of reaching laws (i) (ii) (iii) cannot converge to zero finally. However, under the double power reaching law (iv) proposed in this paper, the convergence rate is improved obviously, and system reaches the sliding surface rapidity and achieves the smooth transition, which can be seen in Figure 6(a). Besides, from Figure 6(b), we can see that its controller output $u$ will have some chattering in the process of reaching sliding plane, but it will decay rapidly as soon as the system state reaches the sliding plane, and the system state will stay near the origin with minimal buffeting.

\section{Conclusion}

An improved double power reaching law of SMC has been investigated in this paper. It was shown that the reaching time was significantly shortened; namely, the dynamic character of the system was greatly improved. Furthermore, the system state was allowed to reach the sliding surface tardily; namely, the transition was smooth. Moreover, the system stayed at the origin eventually, which guaranteed the stability. In a certain external interference, the controller output was ensured to be stable owing to the regulating function of neural network, and the steady-state performance was not affected, which enhanced the robustness of the system.

\section{Acknowledgments}

This work was supported by the National Natural Science Foundation of China (nos. 60904087 and 51109045) and the Fundamental Research Funds for the Central Universities of China (nos. HEUCF110419 and HEUCFX41302).

\section{References}

[1] J. Liu and F. Sun, "Research and development on theory and algorithms of sliding mode control," Control Theory \& Applications, vol. 24, no. 3, pp. 407-418, 2007.

[2] J. K. Liu, MATLAB Simulation for Sliding Mode Control, Tsinghua University Press, Beijing, China, 2005.

[3] W. B. Gao, Y. F. Wang, and A. Homaifa, "Discrete-time variable structure control systems," IEEE Transactions on Industrial Electronics, vol. 42, no. 2, pp. 117-122, 1995.

[4] A. J. Koshkouei and A. S. I. Zinober, "Sliding mode control of discrete-time systems," Journal of Dynamic Systems, Measurement and Control, vol. 122, no. 4, pp. 793-802, 2000.

[5] X. Chen, "Adaptive sliding mode control for discrete-time multi-input multi-output systems," Automatica, vol. 42, no. 3, pp. 427-435, 2006.

[6] K. J. Hunt, D. Sbarbaro, R. Zbikowski, and P. J. Gawthrop, "Neural networks for control systems-a survey," Automatica, vol. 28, no. 6, pp. 1083-1112, 1992.

[7] K. S. Narendra and K. Parthasarathy, "Identification and control of dynamical systems using neural networks," IEEE Transactions on Neural Networks, vol. 1, no. 1, pp. 4-27, 1990.

[8] M. A. Hussain and P. Y. Ho, "Adaptive sliding mode control with neural network based hybrid models," Journal of Process Control, vol. 14, no. 2, pp. 157-176, 2004.

[9] C. H. Tsai, H. Y. Chung, and F. M. Yu, "Neuro-sliding mode control with its applications to seesaw systems," IEEE Transactions on Neural Networks, vol. 15, no. 1, pp. 124-134, 2004.

[10] J. De Jesús Rubio and W. Yu, "A new discrete-time slidingmode control with time-varying gain and neural identification," International Journal of Control, vol. 79, no. 4, pp. 338-348, 2006.

[11] H. Moroika, K. Wada, A. Sabanovic, and K. Jezernik, "Neural network based chattering free sliding mode control," in Proceedings of the 34th SICE Annual Conference, International Session Papers, pp. 1303-1308, IEEE, July 1995.

[12] M. Ertugrul and O. Kaynak, "Neuro sliding mode control of robotic manipulators," Mechatronics, vol. 10, no. 1-2, pp. 239$263,2000$. 
[13] S. Huang, K. Huang, and K. Chiou, "Development and application of a novel radial basis function sliding mode controller," Mechatronics, vol. 13, no. 4, pp. 313-329, 2003.

[14] F. Da, "Decentralized sliding mode adaptive controller design based on fuzzy neural networks for interconnected uncertain nonlinear systems," IEEE Transactions on Neural Networks, vol. 11, no. 6, pp. 1471-1480, 2000.

[15] W. H. Zhang, N. M. Qi, and H. L. Yin, "Neural-network tracking control of space robot based on sliding-mode variable structure," Control Theory \& Applications, vol. 28, no. 9, pp. 1141$1144,2011$.

[16] L. G. Wu and W. X. Zheng, "Passivity-based sliding mode control of uncertain singular time-delay systems," Automatica, vol. 45, no. 9, pp. 2120-2127, 2009.

[17] L. G. Wu and D. W. C. Ho, "Sliding mode control of singular stochastic hybrid systems," Automatica, vol. 46, no. 4, pp. 779783,2010

[18] L. G. Wu, H. J. Gao, and C. H. Wang, "Quasi sliding mode control of differential linear repetitive processes with unknown input disturbance," IEEE Transactions on Industrial Electronics, vol. 58, no. 7, pp. 3059-3068, 2011.

[19] L. G. Wu, P. Shi, and H. J. Gao, "State estimation and slidingmode control of markovian jump singular systems," IEEE Transactions on Automatic Control, vol. 55, no. 5, pp. 1213-1219, 2010.

[20] L. G. WU, W. X. Zheng, and H. J. Gao, "Dissipativity-based sliding mode control of switched stochastic systems," IEEE Transactions on Automatic Control, vol. 58, no. 3, pp. 785-793, 2013.

[21] Q. F. Ren, C. Gao, and P. Wang, "Variable structure control for discrete time systems based on attenuating control," Journal of System Simulation, vol. 20, no. 4, pp. 1056-1059, 2008.

[22] Y. Mi, W. L. Li, and Y. W. Jing, "Variable structure control for a class of discrete-time system based on power reaching law," Control and Decision, vol. 23, no. 6, pp. 643-646, 2008.

[23] B. Sun, X. Sun, L. Chen, and J. Xue, "Algorithm of discrete-time sliding mode control based on power-function," Control and Decision, vol. 26, no. 2, pp. 285-288, 2011.

[24] P. Li, J. Ma, and Z. Q. Zheng, "Sliding mode control approach based on nonlinear integrator," Control Theory \& Applications, vol. 28, no. 5, pp. 619-624, 2011.

[25] H. Mei and Y. Wang, "Fast convergent sliding mode variable structure control of robot," Information and Control, vol. 38, no. 5, pp. 552-557, 2009.

[26] W. J. Li, H. Mei, and Q. J. Li, "A novel sliding mode variable structure control for robot," Machine Tool \& Hydraulics, vol. 37, no. 6, pp. 161-163, 2009.

[27] H. X. Zhang, J. S. Fan, F. Meng, and J. F. Huang, "A new double power reaching law for sliding mode control," Control and Decision, vol. 28, no. 2, pp. 289-293, 2013.

[28] M. J. Wang, G. H. Liu, and F. Wei, "Application of variablestructure control with sliding mode in attitude control of stratospheric airships," Electronics Optics \& Control, vol. 17, no. 6, pp. 65-68, 2010.

[29] L. P. XI, Z. L. Chen, and S. H. Zhang, "Design of sliding mode control scheme based on improved idempotent trending law for robotic manipulators," Computer Measurement \& Control, vol. 20, no. 2, pp. 380-382, 2012.

[30] Y. Niu, D. W. C. Ho, and Z. Wang, "Improved sliding mode control for discrete time systems via reaching law," IET Control Theory and Application, vol. 11, no. 4, pp. 2245-2251, 2010.
[31] C. Gao, Y. Liu, and Y. Li, "A reaching-law method for uncertain discrete variable-structure control systems," Control Theory \& Applications, vol. 26, no. 7, pp. 781-785, 2009.

[32] L. F. Liu, "Application and ordinary solution of a kind of first order differential equation," Journal of HUANGSHI Institute of Technology, vol. 27, no. 4, pp. 41-42, 2011. 


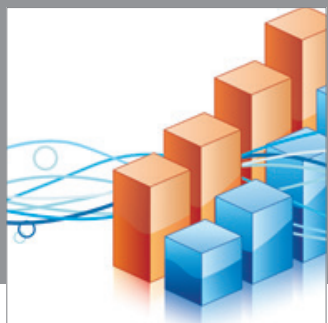

Advances in

Operations Research

mansans

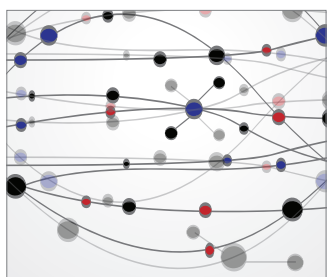

The Scientific World Journal
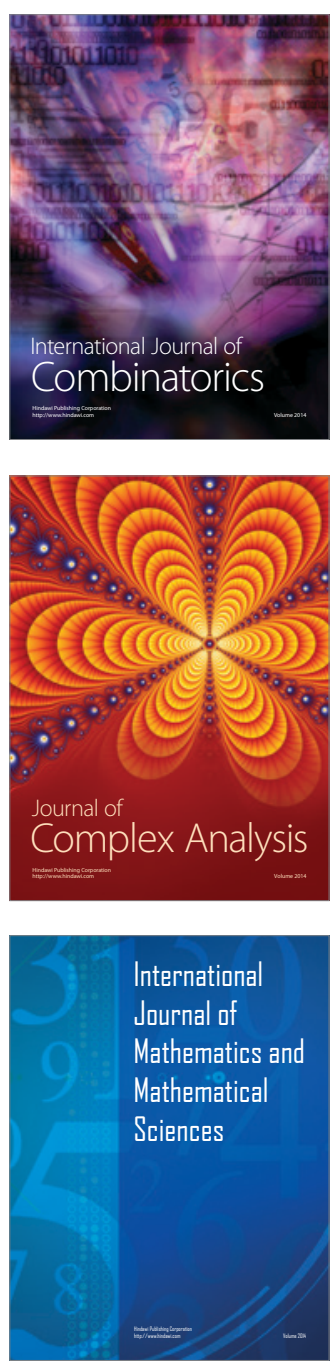
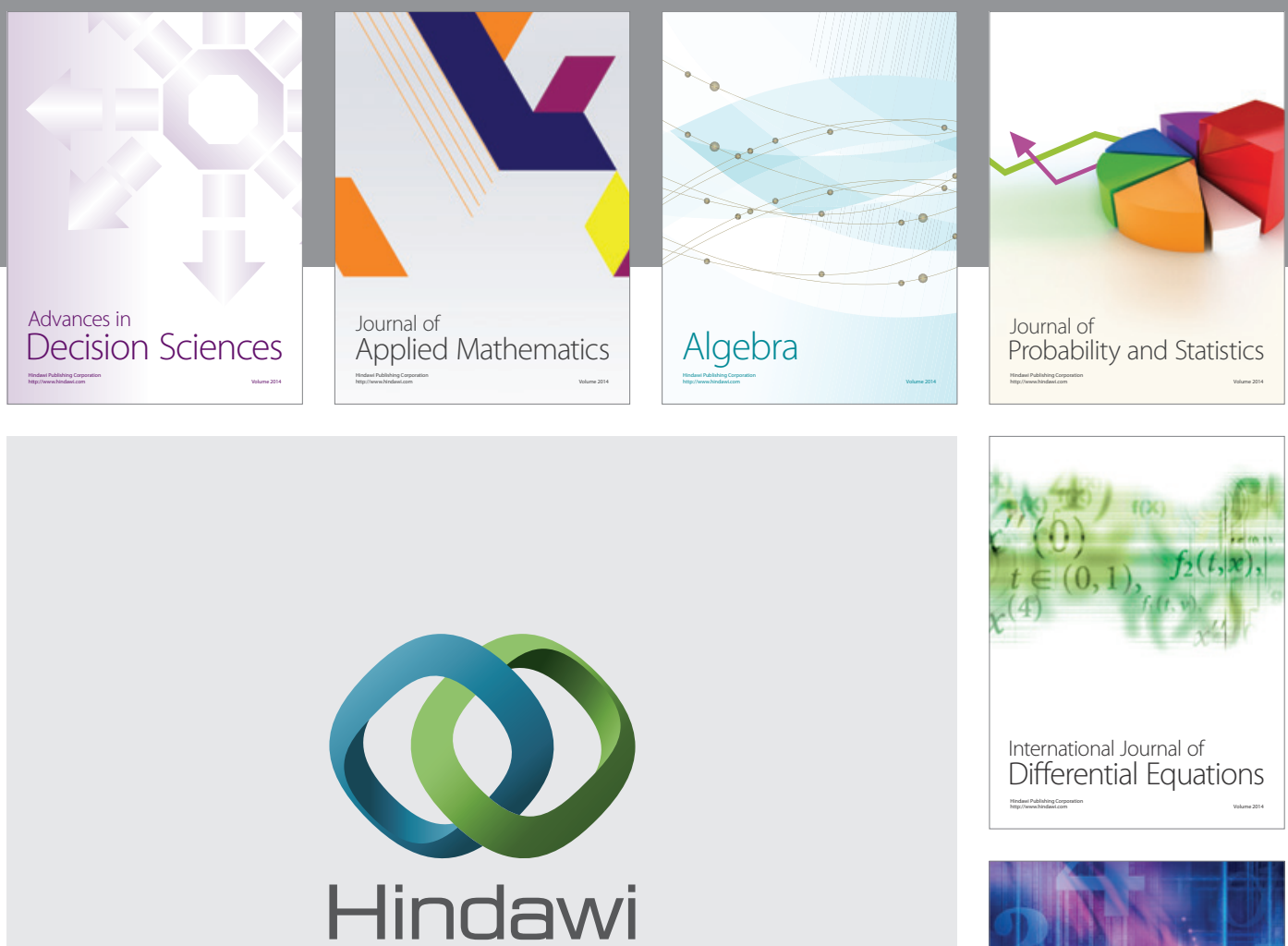

Submit your manuscripts at http://www.hindawi.com
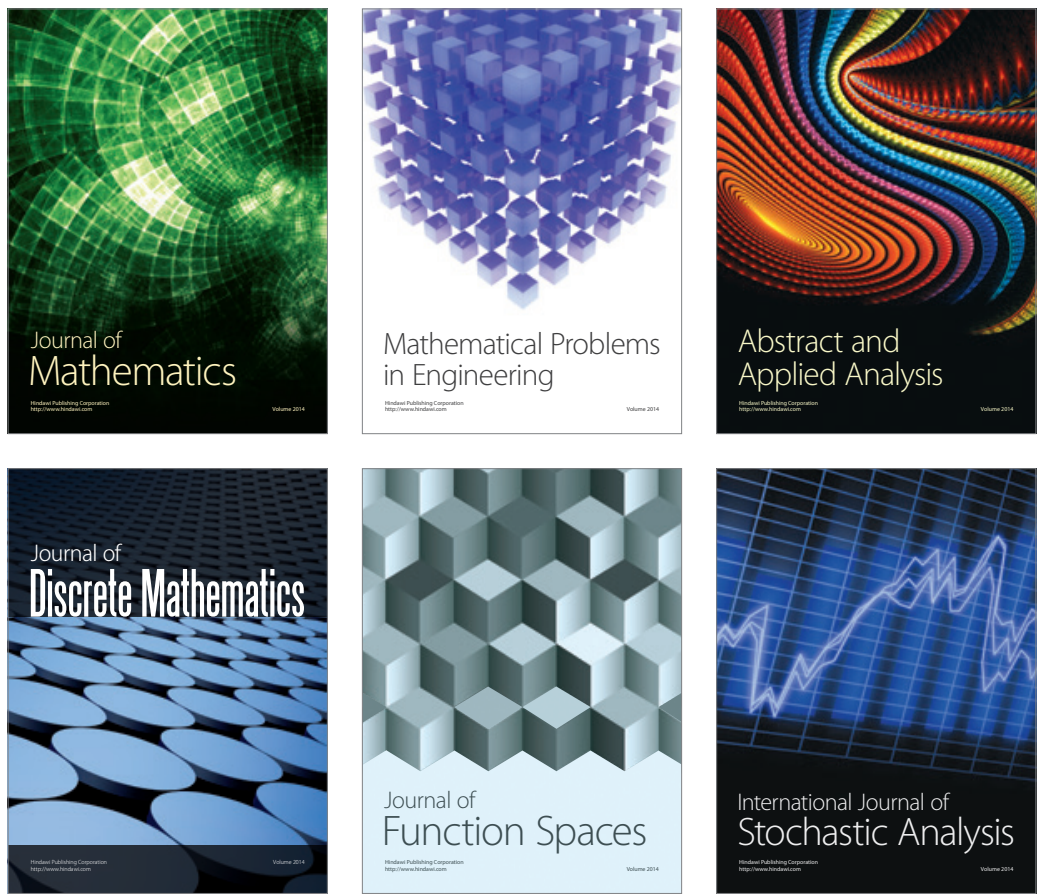

Journal of

Function Spaces

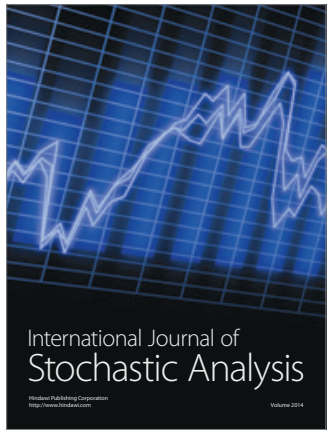

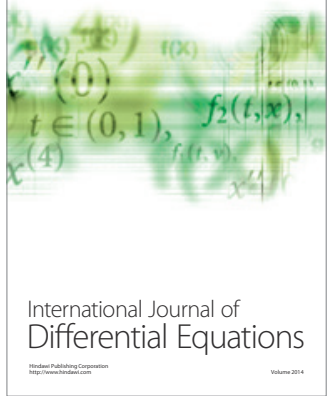
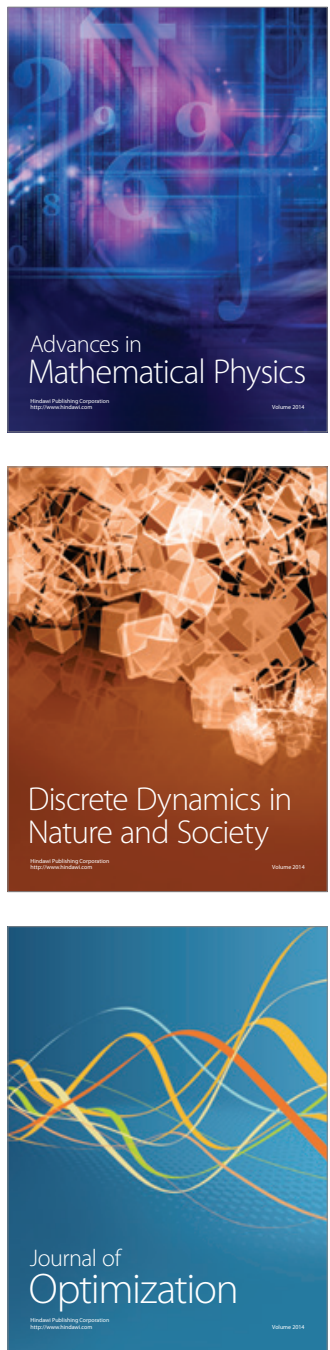